\title{
ARTículo
}

\section{La Jornada Escolar Completa frente a las expectativas de profesionalización de los adolescentes: estudio comparativo de dos colegios de Lima Sur}

\section{All-Day Schooling in the face of teenagers' expectations of professionalization: a comparative study of two schools in southern Lima}

\author{
Diego André López Motta \\ diego.lopez3@unmsm.edu.pe \\ Universidad Nacional Mayor de San Marcos
}

\begin{abstract}
RESUMEN
La Jornada Escolar Completa es una política educativa implementada desde el 2015 para el nivel secundario de la Educación Básica Regular. Incrementa de 35 a 45 las horas lectivas semanales en cursos como Comunicación, Educación para el Trabajo, Inglés y Matemática. Sus objetivos principales son mejorar la calidad de la educación secundaria y promover el cierre de brechas en el Perú. Considerando que todo tipo de proyecto de desarrollo humano debería incluir las expectativas y nociones de futuro de los actores sociales (Appadurai, 2004), este estudio da cuenta de una tensión entre el componente pedagógico de la Jornada Escolar Completa y las aspiraciones profesionales de un grupo de adolescentes que se encuentra cursando el último año escolar.

Palabras clave: Política educativa; Jornada Escolar Completa; adolescencia; expectativas profesionales; transición postsecundaria.
\end{abstract}

\begin{abstract}
All-day schooling is an educational policy implemented since 2015 for the secondary level of the Basic Regular Education. It increases from 35 to 45 the weekly teaching hours in courses such as Communication, Education for Work, English and Mathematics. Its main goals are to improve the quality of secondary education and to promote the closing of wealth gap in Peru. Considering that all kinds of human development projects should include social actors' expectations and notions of the future (Appadurai, 2004), this study accounts for the tension between the pedagogical component of all-day schooling and the professional aspirations of a group of adolescents enrolled in the last year at school.
\end{abstract}

Keywords: Educational policy; all-day schooling; adolescence; professional expectations; post-high school transition.

(C) Los autores. Este artículo es publicado por ISHRA, Revista del Instituto Seminario de Historia Rural Andina de la Facultad de Ciencias Sociales de la Universidad Nacional Mayor de San Marcos. Este es un artículo de acceso abierto, distribuido bajo los términos de la licencia Creative Commons Atribucion - No Comercia Compartir Igual 4.0 Internacional. (http://creativecommons.org/licenses/by-nc-sa/4.0/) que permite el uso no comercial, distribución y reproducción en cualquier medio, siempre que la obra original sea debidamente citada. 


\section{Introducción}

El presente artículo describe los efectos de una política educativa como la Jornada Escolar Completa (JEC) sobre las aspiraciones profesionales de un grupo de escolares urbanos. Por ello, se sitúa entre el fin de la etapa escolar y el inicio de la vida laboral o profesional. Sus principales objetivos son hacer una interpretación antropológica de una política pública para el sector educativo y presentar evidencia etnográfica de las repercusiones de la JEC en los distintos integrantes de la comunidad educativa.

Los datos mostrados son producto de un trabajo de campo con adolescentes de quinto año de secundaria en dos escuelas de Lima Sur: la Institución Educativa (IE) 7100, en San Juan de Miraflores, y la Institución Educativa Emblemática (IEE) Ricardo Palma, en Surquillo. Este esfuerzo por articular comparativamente los elementos que proceden de dos realidades sociales diferenciadas, como lo son el asentamiento humano (AH) en el que se ubica la IE 7100 y la avenida principal de un distrito en la que se encuentra la IEE Ricardo Palma, puede contribuir a percibir la naturaleza de las expectativas profesionales de los actores sociales de una zona de Lima Metropolitana. Evidentemente, estas aspiraciones están condicionadas por factores socioeconómicos, culturales, étnicos, de género, entre otros.

La justificación de este estudio radica en la pertinencia de realizar un balance de la JEC a cinco años de su funcionamiento en algunos colegios públicos del Perú, y la importancia de conocer las voces de los principales implicados en este proyecto pedagógico. Asimismo, la antropología y las ciencias sociales en general rara vez se han mostrado interesadas en el estudio y análisis de la situación educativa a nivel nacional.

Por otra parte, como proponen Golte y León (2011), los jóvenes limeños del siglo XXI pertenecen a una generación importante para estudiar porque sus prácticas pueden marcar a la sociedad incluso en lo político. La adolescencia es el período donde las decisiones pueden estar condicionadas por los problemas individuales, así como por el entorno socioeconómico al que están expuestos los adolescentes.

La investigación de la generación actual es significativa porque dentro de corto tiempo constituirán el conjunto mayoritario de la población nacional (Golte y León, 2011), con la certeza de que serán electores y eventualmente ejecutores. Por eso, la connotación política de sus comportamientos y también de sus imaginarios.

Otro argumento más para un análisis de este tipo es que la disciplina antropológica, hasta el momento, no le ha prestado mucha atención a la capacidad de aspirar, que es un elemento relevante para la cultura (Appadurai, 2004). A través de los imaginarios de los egresados de la formación escolar, se hallará que la realización personal y profesional también es un factor cultural en la sociedad peruana. Es decir, resulta ser un marcador de reconocimiento para las distintas clases sociales, así como para los migrantes de origen andino y sus descendientes que nacieron en la capital.

Primero se efectuará una revisión de las tendencias teóricas y conceptuales sobre temas como la cultura, la adolescencia y la educación. Luego, se elaborará un estado de la cuestión sobre los imaginarios futuros de los adolescentes y, posteriormente, se describirá la metodología empleada. A continuación, se presenta un apartado de la JEC desde una antropología de las políticas públicas. Por último, se exponen los hallazgos de la investigación en distintos subtítulos y las conclusiones. 


\section{Marco teórico y conceptual}

Desde la antropología, los vínculos entre educación, cultura y adolescencia forman parte de la agenda de investigación. Al interior de la tendencia teórica del particularismo histórico, Mead (1962, 1977) realizó estudios comparativos sobre cómo se vivía la personalidad, la sexualidad, la educación y la cultura en Estados Unidos y en sociedades no occidentales como Samoa. Según Mead (1962), los avances acelerados de las invenciones provocan que las nuevas generaciones sean más eficientes que las precedentes. Las tensiones entre los adolescentes y los adultos se refuerzan más cuando se manifiesta el desencuentro entre las viejas y las nuevas formas de vida. La propuesta de la autora es conveniente para el análisis de una sociedad como la peruana, donde la diversificación laboral y, por lo tanto, educativa, ha dado paso a la consolidación de nuevas carreras universitarias y técnicas que tienden a desplazar a las conocidas como tradicionales. En un contexto continental vinculado a la dinámica global, en manos de los adolescentes peruanos del siglo XXI en espacios rurales y urbanos, estos conocimientos, demandados por supuesto por una sociedad en cambios constantes, ocasionan una ruptura generacional.

Un concepto significativo acuñado por Mead(1977) es el de cultura prefigurativa, que consiste en que las nuevas generaciones son las que representan el futuro, recogiendo las influencias de su entorno para adquirir una autoridad frente al mundo. Con este modelo, los aprendizajes pasados se vuelven obsoletos y los adultos no tienen la certeza de cómo se desenvolverán sus hijos en el futuro. El valor de una cultura prefigurativa es predominante en la sociedad peruana, donde los migrantes andinos en Lima impulsan que sus hijos se eduquen, de tal manera que se produce un fenómeno de desvalorización de los propios actores sociales migrantes en relación con las expectativas colocadas en sus hijos nacidos en un contexto urbano. Queda claro que la pérdida de autoridad de los adultos al interior de la cultura prefigurativa posee componentes estructurales y se alimenta de la acción tanto de adultos como de jóvenes.

Al centrar la investigación en los proyectos de vida ligados a la profesionalización de un grupo de adolescentes limeños, se recurre al análisis de la categoría futuro. Appadurai (2004) destaca que el futuro es una dimensión de la cultura que raramente se discute de forma explícita. Según el autor, recuperar esta dimensión tendría efectos concretos para los proyectos de desarrollo.

El presente estudio constata que, en efecto, las expectativas no son incluidas al interior de los proyectos de desarrollo humano, los cuales cobran forma de diseños pedagógicos. La mirada esquiva hacia el futuro es una clara limitación de la antropología. Más adelante se verá cómo la propia noción de voluntad o expectativa se encuentra también estructuralmente condicionada.

Respecto al sistema educativo, Bourdieu y Passeron (1964) plantean que funciona como una suerte de eliminación de las clases sociales más bajas. Además, comprueban que las mujeres de los sectores con menos recursos económicos tienen escasas alternativas para acceder a la enseñanza superior. De acuerdo con estos autores, el reto para una sociología de las desigualdades culturales es contribuir a reducir las desigualdades ante la educación. Su tesis cobra validez para un contexto como el peruano, donde la población más pobre logra estudiar en el colegio durante 6,8 años en promedio, mientras que la población más rica lo hace 12,4 años (INEI, 2018). Es decir, hay una brecha en el acceso a la educación de 5,6 años. Sin embargo, el ideal de la educación persiste, ya que se la imagina como un medio para alcanzar una movilidad social ascendente. En el Perú, esta es una característica de las grandes migraciones andinas a la capital de la segunda mitad del siglo XX, que fue caracterizada por Degregori (1986) como el mito del progreso. 


\section{Estado de la cuestión sobre imaginarios futuros durante la adolescencia}

La adolescencia es un tema que se aborda de manera interdisciplinaria y con distintos enfoques, como por ejemplo su relación con la educación, la transición a la adultez, la precariedad del empleo juvenil, entre otros. Para efectos de este trabajo, se recuperan los estudios que dan cuenta de los imaginarios futuros de los adolescentes peruanos.

Para Rojas y Cussianovich (2013), los jóvenes que están próximos a acabar la secundaria o la han concluido recientemente, se conciben como más independientes y conscientes para tomar decisiones importantes en relación con su bienestar futuro. Las autoras constatan que el discurso de optimismo para conseguir el éxito operaría como un componente psicológico de protección para modificar o reevaluar sus percepciones de bienestar conforme a sus opciones de acción.

Los adolescentes imaginan que la educación postsecundaria significa "vivir bien" y les permitirá "ser alguien" o "ser algo más" en la vida (Rojas y Cussianovich, 2013, p. 43). En ese sentido, se manifiesta una idealización de los estudios superiores, que tienen el fin instrumental de alcanzar la superación o el éxito.

Asimismo, las autoras encuentran que los jóvenes asocian el buen rendimiento escolar con el hecho de que les irá bien en la vida. De esa manera, la preferencia por el estudio configura un imaginario futuro de acceso a la formación universitaria o técnica, aunque en las trayectorias posescolares surjan barreras estructurales.

Guerrero (2014) señala que el ideal de los estudios superiores es un reflejo del gran valor que las familias peruanas le otorgan a la educación. A partir de su investigación con alumnos, padres y docentes, constata que los imaginarios de profesionalización son más frecuentes y el ingreso a la universidad es más viable cuando los adolescentes no forman parte de la primera generación familiar en hacer esta transición.

En un trabajo posterior, Guerrero et al. (2016) evidencian que las aspiraciones de los jóvenes a la educación superior no siempre corresponden a sus posibilidades concretas de ingresar a ese nivel. También exponen que las limitaciones económicas son la barrera más difícil que enfrentan los adolescentes de hogares de bajos ingresos para la materialización de sus pretensiones futuras.

De acuerdo con Guzmán (2016), la educación secundaria genera expectativas y frustraciones entre los estudiantes por ser el último nivel formativo y obligatorio. Entre los resultados de su estudio se destaca que los imaginarios referidos al ámbito universitario son compartidos por los alumnos, sus familias y el personal de la escuela, como tutores y profesores.

Balarin et al. (2017) hallan que los jóvenes limeños que viven en contextos de vulnerabilidad contemplan una estrategia educativa dividida en dos tramos, de los cuales el primero abarca una carrera técnica y el segundo el ingreso a la universidad. Teniendo en cuenta los imaginarios de adolescentes de distritos como San Juan de Lurigancho y Ventanilla, el estudio citado encuentra diferencias según el género:

[...] los hombres aspiran a ser profesionales, aunque por lo general solo consiguen trabajos físicos muy demandantes y que no ofrecen ningún horizonte profesional, mientras que las mujeres anhelan un empleo flexible que puedan compatibilizar con las tareas domésticas y de cuidado (el negocio propio es considerado la mejor opción), pero generalmente solo acceden a trabajos de servicio (Ibidem, p. 76). 
Según la percepción de estos jóvenes, el costo de los estudios superiores es el principal obstáculo para lograr sus propósitos en el futuro. Balarin et al. (2017) aclaran que lo que más influye en las trayectorias de los adolescentes de contextos urbanos vulnerables es la complicación para tener oportunidades disponibles y la carencia de orientación. Así, no se trata solamente de un problema que se limite a la falta de aspiraciones. De manera similar a la presente investigación, la escuela parece no cumplir un papel importante en la toma de decisiones o en la planificación de proyectos de vida, de forma tal que los jóvenes deben decidir por ellos mismos una vez que egresan.

Desde otro ángulo, Urbano (2018) refiere que los adolescentes de un colegio público se trazan la meta de trabajar al concluir la fase escolar para poder cubrir total o parcialmente los gastos de sus estudios superiores. Asimismo, indica que lo hacen por iniciativa propia, corroborando sus deseos de lograr una mayor autonomía.

En resumen, pareciera que los jóvenes peruanos, al formarse imaginarios sobre su futuro, no ponderan las barreras estructurales e idealizan la profesionalización. Esta búsqueda por lograr una identidad a partir de la carrera técnica o universitaria que hayan estudiado, demostraría que en el Perú contemporáneo el éxito es medido por la consecución de logros académicos y laborales.

Otro planteamiento a partir de esta revisión de la literatura es que los imaginarios de futuro son compartidos por la familia y los profesores de la escuela, dando cuenta de la valoración social que se le da a ser alguien. Sin embargo, ambas instituciones, la familia y la escuela, no necesariamente cumplen un papel trascendental en la configuración de aspiraciones, sino que los adolescentes apuestan por una mayor autonomía e independencia. Por otra parte, no debe perderse de vista que los anhelos son influidos por el género. En ese sentido, los varones aspiran a ser profesionales, mientras que las mujeres desean conseguir empleos flexibles para alternarlos con el trabajo doméstico. Esta diferencia de los imaginarios futuros evidenciaría que, desde la adolescencia, las mujeres ya están predispuestas para las labores del hogar y, en efecto, tendrán menos oportunidades de realización que los hombres.

\section{Metodología}

La IE 7100 se fundó el 8 de septiembre de 1985 en el AH Asociación República Democrática Alemana, ubicado en el distrito de San Juan de Miraflores. El inicio de funciones de esta escuela estuvo sustentado con la Resolución Directoral Zonal $N^{\circ} 1700$, emitida durante el gobierno de Alan García. Uno de los motivos principales por los que surgió la institución fue la organización de los vecinos del AH antes señalado, quienes en una asamblea discutieron el problema de la educación de sus hijos. Luego se optó por fundar la IE 7100, que en un principio solo contaba con el nivel primario, inscribiéndose más de 120 niñas y niños. Pertenece a la Unidad de Gestión Educativa Local (UGEL) 01, y en el 2015 el Ministerio de Educación (MINEDU) la incorporó al conjunto de institutos educativos limeños con JEC.

Por su parte, la IEE Ricardo Palma fue creada el 23 de enero de 1948. Inicialmente tuvo su sede en Miraflores y se llamaba Gran Unidad Escolar Tomás Marsano. Tras la Ley $N^{\circ} 12810$ de 1957, que determinaba que los centros educativos debían llevar nombres de peruanos que destacaron como escritores, científicos o héroes nacionales, cambió de nombre a Ricardo Palma. La institución comprendía educación primaria para quinto y sexto grado en la Escuela de $2^{\circ}$ grado Ricardo Palma, educación secundaria completa en el Colegio Nacional Ricardo Palma, Educación Técnica Comercial N 17 Ricardo Palma y Educación Técnica Industrial. Actualmente, pertenece a la UGEL 07 y cuenta con JEC desde el 2015. 
Los criterios por los cuales se seleccionaron estos colegios son principalmente dos. En principio, Lima Metropolitana, debido a su crecimiento demográfico, se ha convertido en un conglomerado urbano multicéntrico, en el que la educación representa un factor considerable en la movilidad social ascendente de sus habitantes. Otra de las razones de esta elección es que la JEC inicialmente fue planificada para colegios con mayor volumen de alumnado, pero tiene limitaciones para expandirse a centros educativos con menor infraestructura (Alcázar, 2016).

En este escenario, surgió la necesidad de comparar las dos escuelas: la IEE Ricardo Palma, que como parte de su diseño histórico preparaba a los alumnos para el mundo laboral, y la IE 7100, cuyo programa curricular no tenía orientación técnica para sus egresados. Ambas instituciones nos ofrecían una perspectiva más equilibrada del panorama educativo en Lima Sur.

La exploración etnográfica duró 8 meses y consistió en visitas permanentes a ambos colegios. Durante la observación del aula, se pudo registrar los discursos de los profesores en el transcurso de las clases, la interacción entre las y los estudiantes, y los mensajes relacionados a las normas de convivencia que estaban pegados en papelógrafos en la pared. Asimismo, se leyeron algunas secciones de los textos escolares oficiales del MINEDU y se corroboró el manejo entre los estudiantes de material publicitario de entidades de educación superior, los cuales fueron entregados en actividades de orientación vocacional.

Los temas que se desarrollaron en las entrevistas a profundidad con los adolescentes incluyeron sus perspectivas de futuro y lo que esperaban al finalizar el período escolar. En total, 20 escolares, 10 por cada colegio ( 5 mujeres y 5 hombres), fueron los participantes del estudio. Además, en la medida que como investigador no tuve acceso a ellos más allá del espacio educativo, las entrevistas abarcaron el empleo de su tiempo libre y qué otras actividades realizaban aparte de estudiar.

Las historias de vida también contribuyeron al ejercicio etnográfico. Una técnica que permitió una aproximación a las trayectorias de las madres de familia fue la que consiste en seguir la vida o biografía, propuesta por Marcus (2001):

La historia de vida, una manera particularmente favorecida de generar datos etnográficos en los últimos años, es un caso especial de seguir la trama. Cómo producir y desarrollar historias de vida como etnografía ha sido un tema de amplias reflexiones, pero pocas veces se ha considerado el uso de la biografía narrativa para diseñar investigación multilocal (p. 121).

Si bien el tema central de la investigación son los efectos de la JEC en la población estudiantil, las historias de vida utilizadas sirvieron para conocer a profundidad los cambios y las permanencias de los migrantes andinos en su desplazamiento a Lima y, principalmente, el rol de la educación en sus aspiraciones y proyectos de vida, tanto de ellos como de sus hijos en el contexto urbano. Lima Sur es el escenario idóneo para la interpretación de sus imaginarios, puesto que en las dos escuelas se halló la presencia de estudiantes que provenían de familias que migraron desde el sur andino.

El análisis de los datos se realizó a través de estrategias descriptivas de carácter etnográfico en las aulas, entrevistas y encuestas a estudiantes, entrevistas con las directoras de los colegios y madres de familia, acopio documental relativo a las escuelas bajo investigación y contraste de los datos recopilados con otros estudios relacionados con el tema. 
La selección de los participantes se realizó según sus niveles de participación e interés mostrado en las distintas materias. Antes del inicio del trabajo de campo, se comunicó a los alumnos de las dos instituciones educativas los objetivos del estudio, la afiliación institucional del investigador, el apoyo que se requería de ellos, la frecuencia de las visitas a las aulas, entre otros aspectos de la investigación. Asimismo, antes de cada entrevista, los participantes fueron consultados sobre su disposición de responder de manera voluntaria algunas preguntas acerca de las carreras de su preferencia, sus proyectos de vida, la enseñanza del colegio y las actividades que realizan más allá del espacio escolar. En todo momento, los padres de familia estuvieron al tanto de la investigación, siendo informados sobre los propósitos y la confidencialidad de los testimonios de sus hijos.

Haber efectuado un estudio comparativo con menores de edad implicó un tratamiento ético de la información y el cuidado de los participantes. Los datos personales de los alumnos de cada colegio fueron proporcionados por el tutor de la sección con la que se trabajó en la IE 7100 y por la auxiliar en la IEE Ricardo Palma, quienes ya tenían conocimiento de los fines de la investigación. Estos datos incluyeron los nombres y apellidos de los estudiantes. Esta información real permitió únicamente una mejor gestión del registro de los entrevistados, teniendo claro que sus nombres no debían ser consignados para salvaguardar su identidad.

Por su parte, las autoridades escolares, desde los directivos hasta los docentes, estuvieron al tanto de las medidas que se tomaron para asegurar el consentimiento informado de los participantes y la protección de su identidad e integridad física. Así, la IEE Ricardo Palma, donde empezó el trabajo etnográfico, estipuló que la totalidad de entrevistas a profundidad con los alumnos se desarrollase en la oficina de la Subdirección General. Para tener uniformidad con el estudio, en la IE 7100, las entrevistas también se realizaron en una oficina de ese tipo, como la Dirección General. De esa manera, en ningún momento el investigador estuvo a solas con los menores. Además, tampoco se los contactó fuera de sus centros educativos.

\section{Una mirada a la JEC desde la antropología de las políticas públicas}

Con la finalidad de comprender de qué manera está diseñada una política educativa como la JEC, se pueden retomar las perspectivas desarrolladas al interior de la antropología de las políticas públicas (Shore y Wright, 1997; Wedel et al. 2005; Huerta, 2013), las cuales ofrecen reflexiones etnográficas sobre el Estado.

Creada el 30 de septiembre de 2014, durante el gobierno de Ollanta Humala y la gestión de Jaime Saavedra como Ministro de Educación, la JEC tiene como objetivos incrementar de 35 a 45 las horas pedagógicas semanales en los colegios seleccionados para las áreas curriculares de Comunicación, Matemática, Inglés y Educación para el Trabajo, promover la equidad educativa, brindar recursos pedagógicos que respondan a los intereses y las necesidades de los escolares, entre otros. De acuerdo con la Resolución Ministerial № 451-2014-MINEDU, la medida se implementó a partir del 2015 en las escuelas públicas con nivel secundario, y su aplicación iba a ser progresiva hasta llegar a 1000 instituciones educativas.

Es posible argumentar que la JEC funciona como un "dispositivo de clasificación" (Shore y Wright, 1997), ya que el alumnado peruano que estudia bajo esa modalidad está siendo preparado con más horas para ciertos cursos, repercutiendo probablemente en mejores oportunidades al concluir la fase escolar. Por el contrario, los estudiantes que pertenecen a colegios con Jornada Escolar Regular (JER) no disponen de las mismas herramientas pedagógicas para su futuro. 
Desde el discurso de la autoridad escolar, se percibe una orientación hacia el ingreso de los adolescentes al campo laboral ni bien cumplan la mayoría de edad. Este es el imaginario de la directora de la IEE Ricardo Palma, para quien un joven representa para el Perú la oportunidad de salir adelante:

Cada persona tiene que ver su realidad y tiene que tener aspiraciones, pero tiene que ver en qué situación está su familia. Yo no puedo exigir a mis padres que me den una profesión. Es más, exigirles todavía que me paguen universidad. Menos. Si el Perú tiene universidades nacionales, yo tengo que ver mi realidad, donde me sale menos los costos. Y lo otro es que ya el chico de 18 años tiene que ser un chico que tiene que entender que tiene que trabajar y estudiar. Ese es el perfil que se quiere de este estudiante. Es la única forma para que el país pueda salir adelante. Un estudiante independiente que se crea y genera su propio trabajo (Directora 1, comunicación personal, 12 de noviembre del 2018).

Una de las intenciones de las políticas públicas es imponer un tipo ideal de lo que debe ser un ciudadano normal (Wedel et al., 2005). Es decir, bajo esta premisa, la directora de un colegio donde funciona la JEC estaría imaginando a los futuros peruanos que reciben esa modalidad educativa como sujetos de producción, con conocimientos técnicos para que puedan autogestionarse ante la falta de oportunidades y el fomento del emprendimiento desde la escuela como mecanismo de movilidad social.

Así, es posible constatar etnográficamente los efectos de la JEC sobre la comunidad educativa. De acuerdo con Wedel et al. (2005), no se trata de preguntar qué es la política, sino qué hace la gente en nombre de la política. La presencia del Estado a través del MINEDU en escuelas con JEC es explícita en ejemplos tan diversos como en el manual sobre el manejo de las tecnologías de la información y comunicación (TIC) (con la JEC, los estudiantes tienen laptops en las aulas desde el 2015), el hecho que las madres lleven el almuerzo a sus hijos o en el discurso de la directora de la IEE Ricardo Palma, que señala que el perfil que busca el colegio en los egresados es que estudien y trabajen.

En línea con lo enunciado por Wolf (1982), la historia se compone de procesos múltiples interconectados, y no es conveniente para las ciencias sociales abordar los fenómenos dinámicos como desconectados o estáticos. Su punto de vista consiste en que las relaciones sociales se mueven simultáneamente en el nivel del sistema general y en el micro-nivel. Así, la JEC responde a un proceso global mayor, a una suerte de receta diseñada desde centros y esferas de poder hegemónico.

Una de las manifestaciones de lo señalado anteriormente podría ubicarse en el impulso que expresa el Banco Mundial (BM) como una de las fuentes externas de financiamiento más importantes para el desarrollo educativo en los países del tercer mundo y como colaborador en la producción de investigaciones en materia educativa (Maldonado, 2000). Cabe recordar que en el Perú esta organización internacional ya ha tenido presencia durante la década de los noventa. Con el apoyo del MINEDU, el BM intentó implementar la creación del bachillerato con el objetivo de establecer un nivel posterior a la secundaria, que no era obligatorio, pero sí un requerimiento para acceder a la educación superior (Guzmán, 2016).

La etnografía en o del sistema mundo, o etnografía multilocal, planteada por Marcus (2001), asume que el objeto de estudio no puede ser explicado en su totalidad si está ubicado en una sola 
localidad, reconociendo la influencia de lo macro y las narrativas del sistema mundo. Asimismo, "construye etnográficamente aspectos del sistema en sí mismo" (p. 112).

En Educación técnica y formación profesional (1992), documento de política del BM, se recomienda el mejoramiento de la calidad de educación secundaria en los países en desarrollo y se manifiesta que las intervenciones adecuadas en ese nivel contribuirían al desarrollo económico y social. Asimismo, el BM promueve mejorar el rendimiento de los estudiantes en matemáticas y ciencias para que comprendan las tecnologías y los procesos de producción modernos.

Esta búsqueda de la calidad, concebida por una esfera hegemónica, coincide con uno de los objetivos de la JEC. Asimismo, la apuesta que hace el BM por las matemáticas es compatible con el aumento de 35 a 45 horas pedagógicas que ha tenido esta área curricular en las escuelas públicas que ahora cuentan con JEC. En efecto, una sugerencia de este tipo moviliza a los países en desarrollo que, como el Perú, han implementado estrategias educativas para la mejora de la calidad en el nivel secundario.

Más que lograr el desarrollo a través de intervenciones pedagógicas, la aplicación de la JEC correspondería a una visión desarrollista de la educación. Nandy (2011) sostiene que el desarrollismo ocasiona que los sectores más débiles de una sociedad paguen un costo muy alto por el desarrollo. Si el Perú apunta a lograr una modernización productiva y competir con éxito en el mercado internacional, es a costa de ciertas desventajas en la formación de los adolescentes que actualmente estudian en escuelas con JEC, como no tener qué y dónde almorzar. Durante el trabajo de campo, se observó que de 12:45 p.m. a 01:15 p.m., hora de refrigerio implementada por la JEC, algunos padres de los escolares de la IE 7100 no llevaban el almuerzo a tiempo y otros no lo llevaban. Esto generaba que algunos estudiantes tuvieran que compartir su almuerzo. Asimismo, los estudiantes que recibían su refrigerio tarde se lo tenían que llevar al salón para consumirlo ahí mismo. En cuanto a infraestructura, el colegio no dispone de comedores suficientes y se observó que los escolares almorzaban sentados al borde del patio principal.

El documento que sienta las bases de la presencia del BM en el sector educativo de los países en desarrollo es Prioridades y estrategias para la educación. Examen del Banco Mundial (1996). La entidad expresa que su apoyo es fundamentalmente con asesoría especializada:

El Banco Mundial está firmemente decidido a seguir dando apoyo a la educación. Sin embargo, aunque el financiamiento del Banco equivale actualmente a la cuarta parte de toda la ayuda para la educación, este financiamiento sigue siendo todavía menos del $0,5 \%$ del gasto total de los países en desarrollo en el sector. Así pues, la principal contribución del Banco debe consistir en el asesoramiento destinado a ayudar a los gobiernos a elaborar políticas de educación adecuadas a las circunstancias de sus países. El financiamiento del Banco se diseñará generalmente para fomentar el gasto y el cambio de las políticas por parte de las autoridades nacionales (p. 17).

De acuerdo con Maldonado (2000), las pautas del BM están más enfocadas en la educación básica porque sus tasas de rentabilidad social son mayores que las de la educación superior. En ese sentido, el proyecto de esta organización en términos educativos apunta a una lógica de inversión y productividad, en otras palabras, de costo y beneficio. Es esta naturaleza corporativa la que se inscribe en las políticas educativas nacionales de los países en los que el BM tiene injerencia. 
El informe Ninis en América Latina. 20 millones de jóvenes en busca de oportunidades (2016), elaborado por el BM, sugiere como opción de política para los países de la región, la transición a escuelas de tiempo completo, con el fin de evitar la deserción escolar, factor que provoca que los jóvenes no estudien y estén desempleados. En ese orden de ideas, la JEC también tendría los objetivos adicionales de asegurar la permanencia en el colegio y prevenir el aumento de ninis que, según el BM, representan el 10,9\% de los adolescentes en el Perú.

Para Huerta (2013), cuando los Estados se alinean con los programas internacionales no siempre se deriva en una política pública exitosa. Por más que las entidades globales como el BM ofrezcan la receta, las realidades de cada país son distintas y las particularidades de sus ciudadanos también. En el campo de la educación, esto es más sensible porque se generan brechas entre los mismos estudiantes.

Lógicamente si tú no comes hasta las 03:30 p.m. tu salud va a estar afectada. Se pensó en un principio que la JEC iba a ser beneficiada con alimentos también, pero hasta ahora nada. Entonces hay chicos que almuerzan y hay chicos que no almuerzan. Y con todo el tema de salud que puede acarrear esto: gastritis, anemia, TBC (tuberculosis) (Directora 2, directora de la IE 7100, comunicación personal, 23 de noviembre del 2018).

La revisión documental del BM que se ha presentado demostraría que la JEC es una política peruana inmersa en el sistema mundo, movilizada por recomendaciones que se construyen desde centros de poder. Si bien el trabajo de campo permite visibilizar sus efectos, el análisis documental desvela su origen. "En las políticas públicas están en juego el control por los recursos económicos, pero para tener el control de dichos recursos, es necesario tener el control de esas metáforas clave que configuran la realidad social" (Huerta, 2013, p. 5).

Una de las justificaciones para dar paso a este tipo de políticas educativas tiene un fundamento real: la ubicación de los escolares peruanos en las posiciones casi últimas en los rankings internacionales de evaluación educativa. Esto valida la implementación de los proyectos pedagógicos entre los líderes políticos y la propia sociedad civil. Alcázar (2016) sostiene que la JEC se llevó a cabo a partir de los bajos resultados en la prueba PISA (Programa Internacional para la Evaluación de Estudiantes), donde el Perú ocupó el último lugar de 65 países evaluados en el 2012.

\section{Expectativas diferenciadas en Surquillo y San Juan de Miraflores}

Un primer hallazgo fue que los participantes del estudio en la IEE Ricardo Palma no están interesados en la educación superior técnica, mientras que ése parece ser el camino de los egresados de la IE 7100. En tal orden de ideas, el perfil de las alumnas y los alumnos de la IEE Ricardo Palma, difiere del de los egresados de la IE 7100. Sin embargo, en ambos casos los imaginarios profesionales se consideran mecanismos de movilidad social, que no necesariamente son compatibles entre sí, a pesar de la naturaleza pública de las dos instituciones educativas. Cabe destacar que los que prefieren la educación técnica, principalmente los adolescentes de San Juan de Miraflores, conciben ese tipo de formación como el medio inicial para una instancia universitaria posterior.

Esto evidencia una continuidad en el imaginario social peruano: que ser profesional universitario es la única vía para lograr el reconocimiento, aunque no repercuta necesariamente en una movilidad social. Si bien hay precariedad laboral y tasas altas de desempleo de profesionales, los títulos conseguidos gracias a los estudios universitarios siguen ejerciendo una función de valoración social en el Perú contemporáneo. 
Al respecto, Bonfiglio (2008) encuentra que las intenciones de estudiar en una universidad son mayoritarias en comparación con los institutos de formación superior en todos los niveles socioeconómicos. Las principales razones son: la baja calidad de la enseñanza, la infraestructura deficiente y el equipamiento obsoleto de los institutos tecnológicos públicos. El autor también sostiene que los estudiantes de carreras técnicas consideran que la institución donde están matriculados tiene poco prestigio e ingresaron de manera resignada, ya que su objetivo primario fue la universidad.

En efecto, Bonfiglio señala que en el Perú la universidad sigue siendo hegemónica en el nivel de instrucción superior: "[...] en el sistema educativo peruano no hay un camino alternativo a la universidad, que signifique un nivel de profesionalización que goce de suficiente aceptación y prestigio" (Bonfiglio, 2008, p. 81). A fin de ubicar en el tiempo una posible explicación a esta tendencia de la educación peruana, describe 2 hitos principales sobre la formación técnica en el siglo XX.

El primero fue la disolución de la Escuela Nacional de Ingenieros Técnicos (ENIT) en la década de 1960. La organización se fundó con el propósito de ofrecer carreras cortas a egresados de secundaria que no lograban ingresar a la universidad. No obstante, los estudiantes y egresados no se conformaron con obtener un título de carrera corta, ejerciendo presión para conseguir el título universitario. Con movilizaciones estudiantiles y tomas de locales, finalmente lograron su propósito, y la Universidad Nacional de Ingeniería (UNI) los admitió, para posteriormente alcanzar el título de ingenieros.

Si bien la lucha de los estudiantes de la ENIT tenía propósitos educativos, en el fondo tenían también un carácter social, en la medida en que en su gran mayoría formaban parte de un contingente de migrantes o hijos de migrantes que pugnaba por ascender socialmente. En realidad, el reclamo por acceder al título de "ingeniero" formaba parte de la búsqueda de ascenso social de estos sectores emergentes (Bonfiglio, 2008, p. 86).

Así, a partir de la segunda mitad del siglo XX, la educación y subsiguiente profesionalización han sido, para los migrantes a la capital, un mecanismo para distinguirse y alcanzar cierta reputación. Eso representa una permanencia en el imaginario de los migrantes y de sus hijos, quienes siguen valorando más a la universidad y apuntan a estudiar en ella en algún punto de sus trayectorias.

El segundo hito fue el fracaso de las Escuelas Superiores de Educación Profesional (ESEP) en la década de los setenta. Estas entidades fueron creadas para pertenecer a un nivel posterior a la educación básica, con el objetivo de brindar una formación y certificación para los egresados, que luego podrían insertarse en el mercado laboral sin estudiar en la universidad. La dificultad que tuvo este programa educativo fue la resistencia social, especialmente de enfermeras y maestros. Estos trabajadores calificaron sus carreras como universitarias y rechazaron la reducción de esa condición a nivel de las ESEP, que solo tenían la capacidad de otorgar una carrera corta y emitir títulos intermedios (Bonfiglio, 2008).

El autor explica que la entrada de la formación técnica al nivel superior es el resultado de un proceso histórico registrado durante el siglo XX, período en el que los sectores sociales emergentes han entendido a la instrucción superior solo en términos de universidad y, por lo tanto, han ejercido presión para acceder a ella, buscando el ascenso social.

En este recuento es perceptible la incapacidad del Estado para evitar la presión social ascendente, lo que lo ha llevado a incorporar a la formación técnica dentro de los estudios superiores, 
de acuerdo con Bonfiglio. Al mismo tiempo, se mantuvo la gratuidad de la universidad pública, teniendo como efecto el rechazo de la formación técnica, pues los egresados de secundaria solo optaban por estudios universitarios.

\section{Límites y posibilidades de la JEC en la transición postsecundaria}

En línea con lo expuesto por Rojas y Cussianovich (2013), al término del nivel secundario, un adecuado rendimiento escolar se manifestaría en un futuro éxito tanto en el campo académico como en el profesional. Por ello, los estudiantes se preocupan por comprender las materias que están relacionadas con sus objetivos inmediatos. Si bien la JEC ha incrementado las horas de dictado para Matemática, esa área curricular genera más desorientación que certezas en el aprendizaje. Es lo que transmitieron las estudiantes de la IEE Ricardo Palma en relación a si se sienten preparadas académicamente para la educación superior:

No tanto porque en Matemática debería haber un poco más de temas, porque cada año se repite el mismo tema. Eso es lo que me he dado cuenta. Falta todavía un poco (Estudiante 1, comunicación personal, 10 de julio del 2018).

Para ella, los contenidos de un curso que considera elemental como Matemática presentan una continuidad de año en año, lo que no repercutirá en su desenvolvimiento posterior para tentar estudios superiores. La entrevistada planea seguir carreras como Ingeniería Industrial, Psicología o Educación, y demanda una diversidad de temas que tengan concordancia con espacios más allá de la escuela, a partir de la identificación de un mismo programa curricular año tras año.

Sí, pero siento que en Matemática todavía estamos en lo básico, me gustaría aprender un poco más. Siento que nos enseñan muy poco para el examen de admisión de alguna universidad. Aún estamos en Pitágoras y me gustaría que enseñen un poquito más porque en esos tests de universidad que yo he visto por internet, es un poquito más difícil matemática y siento que es muy poco lo que enseñan aquí (Estudiante 2, comunicación personal, 10 de julio del 2018).

La comparación entre la enseñanza del colegio y lo que se solicita para ingresar a la universidad muestra un abismo notorio que termina por influir en la valoración que los alumnos les dan a sus capacidades. Sienten encontrarse en un nivel básico del cual buscan salir pensando en los resultados posteriores. El aprendizaje de Matemática tiene un propósito completamente instrumental e inmediato que es el ingreso a la universidad, pues la adolescente está pensando estudiar Psicología o Derecho, carreras donde ese curso no es significativo. Contraponer lo básico a lo difícil, lo elemental a lo avanzado, en suma, la dicotomía colegio-universidad, revela que algunos conocimientos que se adquieren en quinto de secundaria se vuelven obsoletos porque no sirven a cuestiones prácticas como aprobar un examen de admisión.

En cambio, en la IE 7100 los estudiantes sí notan que están siendo preparados para la educación superior. Más que en la forma de dictado de cursos, se pone énfasis en la responsabilidad del alumno, quien debe aprender por su cuenta:

Sí, está bien porque nos enseñan bien. Lo que pasa es que los alumnos a veces no hacemos caso al profesor y ese es el problema también. Pero enseñan bien en el colegio (Estudiante 3, comunicación personal, 23 de noviembre del 2018).

Asimismo, las entrevistas a profundidad con estudiantes que están ahí desde el inicio de la secundaria han servido para conocer cómo a lo largo de ese nivel la educación ha mejorado: 
Creo que estos últimos años me está preparando mejor que toda la secundaria que he estudiado aquí. Ahora en quinto ha mejorado más la educación. En la materia de Matemática han traído a un profesor que ahora nos ha hecho renovar más el aprendizaje. Nos está enseñando cosas nuevas que no habíamos aprendido desde primero de secundaria (Estudiante 4, comunicación personal, 30 de noviembre del 2018).

Nuevamente, el curso de Matemática es relevante para los egresados de los colegios en estudio. La diferencia es que en la IE 7100 se afirma que hay una buena enseñanza y no se demanda una actualización de sus contenidos. De esa manera, se encuentra que la apuesta de la JEC por Matemática tiene efectos distintos en dos instituciones de una misma zona urbana.

\section{Algunas diferencias individuales en las oportunidades hacia el futuro}

Se constató que entre las adolescentes de ambos distritos hay ciertas diferencias. La estudiante 5 vive en Surquillo y en el 2018 finalizó sus estudios en la IEE Ricardo Palma, teniendo en mente estudiar Arquitectura y Derecho en una universidad. Por su parte, la estudiante 6 vive en Villa María del Triunfo y egresó de la IE 7100 también en el 2018. Ella contemplaba estudiar Contabilidad o Administración Bancaria en un instituto. Estando en la misma situación de egresadas de la Educación Básica Regular y con aspiraciones de profesionalización, las disparidades entre ellas se reflejan en las actividades que hacen los fines de semana.

Todos los domingos de 9 a. m. a 1 p. m. me voy a mis clases de Excel que tengo por San Miguel. La verdad es que más me he metido porque mi mamá me ha dicho que tengo que entrar a la universidad con conocimientos. Tengo una amiga que es universitaria y también está llevando ese curso de Excel porque necesita (Estudiante 5, comunicación personal, 21 de agosto del 2018).

Sus padres la matricularon en ese curso porque lo ven como una herramienta si accede a los estudios superiores. El uso del tiempo de los días domingos es considerado en su hogar como útil y ella tiene la oportunidad de sacarle provecho y llegar con más preparación a la vida universitaria. Por su parte, la estudiante 6 suele hacer otra actividad ese mismo día: "Los domingos trabajo en venta de pescado con una señora de 7 a. m. a 2 ó 3 p. m. De ahí descanso o llamo a mi mamá" (Estudiante 6, comunicación personal, 30 de noviembre del 2018).

Con ambos testimonios puede verse la diversidad de mujeres que egresaron de la educación básica en el 2018. La estudiante 5 está preparándose para la universidad con un curso y la estudiante 6 tiene que trabajar los domingos. En ese sentido, si bien ambas inician la transición postsecundaria, desde ya se observa que la primera está teniendo más herramientas para llegar preparada a los estudios superiores. Para ella, el uso de Excel le servirá para su carrera de Arquitectura o Derecho, es decir, una trayectoria universitaria que le podría dar más ventajas para lograr una movilidad social ascendente. En cambio, el trabajo manual que hace la estudiante 6 todos los domingos tiene poca valoración social y no le ofrece beneficios para insertarse satisfactoriamente a los estudios superiores.

Aparte de la diferenciación entre las dos según lo que hacen en su tiempo fuera del colegio, hay diferencia con respecto al origen de los padres que podrían explicar la situación de las estudiantes. Los padres de la estudiante 5 nacieron en Lima, mientras que los padres de la estudiante 6 son originarios del anexo de Accoquipa, distrito de Sara Sara, provincia del Páucar del Sara Sara, Ayacucho. Su migración a Lima puede ser interpretada como el "mito del progreso", expresión acuñada por Degregori (1986) que refiere que los pobladores andinos llegan 
a las ciudades para lograr un futuro mejor a través de la educación, el trabajo asalariado y el comercio.

Utilizando la técnica de Marcus (2001) de seguir la vida o biografía, se halló que los padres de la estudiante 6 se mudaron a Jicamarca, en San Juan de Lurigancho, porque inauguraron su negocio propio de panadería. En efecto, su hija asumió los roles de la casa y se despertaba más temprano para preparar la comida que ella y su hermano llevaban al colegio. Aunque la estudiante 5 también se hacía cargo de su hermano recogiéndolo del colegio, dispone del tiempo y del apoyo de sus padres para estudiar los domingos. De esa manera, las oportunidades de ambas mujeres difieren, pudiendo repercutir en su transición postsecundaria.

\section{El rol de las madres de familia}

Las madres de los adolescentes ofrecieron una perspectiva de esta transición desde el hogar. En ese sentido, se pudo conocer cómo es el proceso de acompañamiento en este período previo a la adultez y a los estudios superiores. Sus discursos evidencian que, entre las familias de Lima Sur, se ponderan diferentes factores respecto a las trayectorias futuras de los hijos.

Siguiendo la vida de la madre 1, su padre la trajo a Lima cuando tenía 4 años desde Corculla, distrito de la provincia del Páucar del Sara Sara, en Ayacucho. No pudo concluir la educación básica porque vivió en un entorno violento por su madrastra y se quedó en tercero de secundaria. Actualmente, cuida a una adulta mayor y los fines de semana trabaja con otro paciente. A su parecer, la finalización del período escolar de su hijo es un alivio:

Antes, cuando yo trabajaba a medio tiempo, se iba temprano a la casa a almorzar, pero ahora hay problemas con el almuerzo del colegio, no hay comedor. Al principio dijeron que sí iba a haber un comedor, que no se preocupen. Pero hasta ahora no hay un comedor. Antes las mamás tenían que traer la comida, la dejaban en la puerta. Después prohibieron eso. Ahora los chicos tampoco no pueden traer porque comen la comida toda fría. Es muy complicado. Ya no veo los días que acabe el colegio. Yo tengo muchos problemas con el almuerzo porque él está bien delgado, me da miedo que se enferme (Madre 1, comunicación personal, 12 de octubre del 2018).

La JEC es un problema para ella debido a la dificultad que implica para el almuerzo de su hijo; de ahí sus deseos de que egrese pronto. Un testimonio como el suyo representa la transformación que esa política educativa ha generado en los hogares peruanos.

La madre 2 migró a Lima desde la comunidad de Incaraccay, distrito de Cangallo, provincia del mismo nombre, en Ayacucho. No pudo concluir la educación básica porque en su escuela desaparecieron a los profesores y a otros los llevaron presos durante el conflicto armado interno. En consecuencia, se quedó en primero de secundaria. Actualmente, trabaja limpiando casas y a veces lleva a su hijo para que la ayude. Así se refiere al momento que atraviesa el menor:

Él está preocupado, a la vez quiere trabajar. Está indeciso. No sabe qué estudiar. Así está preocupado. A veces trato de hablarle que primero tiene que estudiar. Tiene que estudiar porque está jovencito y mejor que aproveche mientras hay posibilidad (Madre 2, comunicación personal, 8 de enero del 2019).

Sin embargo, el adolescente no expresó la opción de trabajar durante la entrevista. De tal manera que al concluir la etapa escolar tiene dos caminos y, por lo tanto, está indeciso. Es su madre la que quiere que estudie, pues cree que en el hogar hay la posibilidad de ofrecerle 
una formación posterior al colegio. En efecto, su rol es convencerlo de que la prioridad son los estudios y no el trabajo.

La madre 3 proviene de Coracora, capital de la provincia de Parinacochas, en Ayacucho. Estudió el nivel secundario en Lima y luego siguió Computación como formación superior, pero no lo pudo terminar por su embarazo. Ahora es ama de casa y durante el período escolar iba todos los días a la IE 7100 a dejarle el almuerzo a su hijo en la hora de refrigerio. En relación a la transición posescolar del menor, ella y su pareja están dispuestos a apoyarlo en lo que decida estudiar. Pero lo que más le preocupa es que su hijo se equivoque al elegir la carrera de Mecánica Automotriz, considerando el dinero y el tiempo que se destinarán para ello.

A veces yo me pongo a pensar: de repente quiere estudiar Mecánica por estar junto con su primo. Es un cambio medio raro porque al comienzo me dijo que no porque se ensucia. A veces se encuentra con su primo y yo no quiero. Es como un presentimiento (Madre 3, comunicación personal, 18 de diciembre del 2018).

Según ella, su hijo quiere estudiar Mecánica Automotriz por injerencia de su primo, ya que recuerda que al inicio no le gustaba porque se ensucia. Tras concluir la etapa escolar, aunque el interés vocacional haya sido influido por un integrante de la familia no nuclear, los padres tienen la función de asegurarse si esa decisión es la correcta. Asimismo, como expresa la madre 3, la interacción del adolescente con su primo consolidaría su elección. En ese orden de ideas, el apoyo de los padres se da si los hijos defienden con argumentos más sólidos su vocación y no se basan solo en lo que vieron en otros integrantes de la familia.

En otra línea, es importante resaltar la función que tiene la educación para las migrantes andinas en la capital y para la materialización de los proyectos familiares. El hijo de la madre 3 nunca ha vivido con su padre y ahora la pareja de ella se hace cargo tanto del menor como de su hermano.

Él conoce a mi pareja actual de pequeñito. Entonces prácticamente ha convivido toda la vida ahora con mi pareja actual. A su papá ni lo conoce. Una vez se encontró de casualidad cuando fuimos al parque y su papá ni lo abrazó, nada. Mi hijo sintió la emoción, lloró, lo abrazó. A pesar de todo yo no le digo a él que le tenga cólera (Madre 3, comunicación personal, 18 de diciembre del 2018).

Según Anderson (1990), la reconstitución de relaciones de pareja en mujeres migrantes urbanas se trataría de una estrategia económica, es decir, tener con quién compartir la lucha por sacar adelante a los hijos. Esta característica se hace más visible en el último año escolar de los menores, puesto que las madres y los padres les dan su apoyo no solo en términos emocionales, sino también en el plano económico.

En contraste, se nota que a una madre de un adolescente que estudia en la IEE Ricardo Palma le ha afectado la implementación de la JEC en cuanto al almuerzo de su hijo. Por ello, interpreta que la fase escolar ha representado una carga y una preocupación para ella, sobre todo en la alimentación del estudiante.

Las madres de familia entrevistadas en la IE 7100 han considerado otros aspectos para el momento en el que se encuentran sus hijos. Su futuro, una elección segura de la carrera a seguir y la tensión entre trabajar o estudiar, fueron los temas que más merecieron la atención de las madres. Pareciera que ya han asimilado una modalidad de estudios como la JEC, pues no han mencionado ninguna crítica, como sí lo ha hecho la madre de un alumno de la IEE Ricardo 
Palma. Por el contrario, la madre 3 ya se adaptó y todos los días iba al colegio a dejarle el almuerzo a su hijo.

\section{El capital social en los proyectos de vida}

Otro de los resultados de esta investigación es que la mayoría de los alumnos de la IEE Ricardo Palma nunca ha trabajado y la mayoría de los de la IE 7100, sí. En el primer colegio, solo una participante del estudio, que vive en Villa El Salvador, ha trabajado vendiendo ropa y cremoladas. En el segundo, los trabajos mencionados fueron: lavado de autos en un taller y ayudante en una carpintería; "algunos cachuelos para hacer plata"; vendedora en la tienda de mueblería del primo por Plaza Norte; ayudante en la panadería del padrastro en los meses de vacaciones; cargador de arena y ladrillo para que los vecinos construyan sus casas; vendedora de pescado; ayudante en la limpieza del hogar de las vecinas o cuidando a sus bebés; y ayudante del papá con los materiales en las obras de construcción y de la mamá cuando va a limpiar casas.

Las características de estos trabajos concuerdan con la literatura: los hombres consiguen trabajos físicos demandantes sin horizonte profesional y las mujeres tienen trabajos orientados al servicio (Balarin et al., 2017), y la mayoría de adolescentes peruanos no cuenta con beneficios laborales y se desenvuelven en la informalidad (Franco y Nopo, 2018).

Se afirma que los estudiantes que viven en distritos como Villa El Salvador, Villa María del Triunfo y San Juan de Miraflores no esperan cumplir la mayoría de edad para obtener ingresos, mientras que los de Surquillo solo se dedican a las tareas escolares y otras actividades. Al ver que son el padre, el padrastro, la madre, los primos, las vecinas y los vecinos quienes les aseguran un trabajo a los jóvenes de la IE 7100, se ha hallado una permanencia de lo que ya han señalado las investigaciones de antropología urbana en el Perú (Degregori, Blondet y Lynch, 1986; Golte y Adams, 1987): la importancia de las redes familiares y vecinales para mantenerse en una ciudad como Lima. La mayoría de padres y madres de estos adolescentes no nacieron en la capital $\mathrm{y}$ en sus respectivas familias siguen reproduciendo lazos para garantizar puestos laborales o iniciativas de emprendimiento.

Degregori et al. (1986) interpretan que estas prácticas suponen "la cerrazón del triángulo sin base" (p. 158), modelo propuesto por Cotler (1968) para explicar la casi ausencia de relaciones horizontales en el Perú, sustituyéndolas por relaciones verticales de dependencia. Para los autores, "el de abajo ya no está solo, ha tejido con otros su red de relaciones 'vecinales' que le permiten convertirse en "poblador"” (Degregori et al., 1986, p. 158). Las nuevas generaciones siguen reproduciendo estas estrategias y encuentran en el trabajo para los vecinos, como cuidar a sus hijos o ayudarlos a construir sus casas, la oportunidad para recibir cierto tipo de ingreso económico y así ya no depender de sus padres.

Para los migrantes de segunda generación, la constitución de redes también influye en su imaginario posescolar, sobre todo de los varones. Por ejemplo, el papá del estudiante 3 , que es albañil, al trabajar con su hijo en construcción, es posible que haya fomentado en él el interés por la Ingeniería Civil, carrera que quiere estudiar. A su turno, otro adolescente, al frecuentar un taller de autos con su primo y haber trabajado en el lavado de los mismos, se ha interesado por la Mecánica Automotriz.

En la IEE Ricardo Palma, como parte de Educación para el Trabajo (EPT), se enseñan los cursos de Computación e Informática, Mecánica Automotriz, Contabilidad, Electricidad, Electrónica, Industria del Vestido, Industria Alimentaria, Carpintería y Ebanistería, Mecánica de Producción y Construcciones Metálicas. Si la IE 7100 tuviera estas mismas materias, estas serían 
mejor aprovechadas por el alumnado de quinto de secundaria. Sin embargo, los estudiantes no ven como primera opción laborar en algo para lo que están siendo formados, pues sus anhelos profesionales coinciden con la formación en EPT. En esta escuela, algunas de las preferencias vocacionales fueron Contabilidad, Industrias Alimentarias, Gastronomía, Mecánica Automotriz y Electricidad Industrial. En efecto, se produce una incompatibilidad entre los intereses de los futuros egresados y los recursos que la escuela pone a su disposición para garantizarles opciones laborales inmediatas. Así, la JEC es una política educativa que no tiene en cuenta las aspiraciones profesionales ni las realidades socioeconómicas de este grupo de estudiantes, pero sí el imaginario de que la formación técnica los podría conducir a su inserción laboral.

De acuerdo con Appadurai (2004), los proyectos de desarrollo deben identificar las aspiraciones de las personas en su intervención. Si en la IE 7100 los estudiantes solo llevan un curso de EPT, Computación de Informática, durante toda la secundaria, en comparación con los de la IEE Ricardo Palma, quienes son instruidos en 10 cursos, estarían perdiendo la oportunidad de prepararse mejor desde el colegio en algo que sí han decidido ejercer en el futuro.

No obstante, en la IE 7100 los egresados han definido sus horizontes profesionales no por los conocimientos obtenidos en la escuela, sino en el aprendizaje que logran a través de la interacción con sus redes familiares. Son los casos del alumno que seguirá Mecánica Automotriz por haber estado presente en el taller con su primo; el estudiante 3, que se animó por Ingeniería Civil para ayudar a su padre que es albañil; y otro escolar, que decidió estudiar Electricidad Industrial porque su hermano mayor y su cuñado son electricistas.

En estos ejemplos, es perceptible la permanencia de un sector de adolescentes de Lima Sur con sus familiares. Sin ello, no hubieran elegido ciertas tendencias profesionales o no hubieran desarrollado sus vocaciones. Por lo tanto, resulta sustancial el tiempo no escolar, aquel que transcurre fuera de las aulas, como una oportunidad para autoformarse. Si bien uno de los objetivos indirectos de la JEC es prevenir situaciones de riesgo para los jóvenes, como los embarazos no deseados y que no incurran en la delincuencia, también sería indispensable reconocer que ese tiempo y ese espacio más allá del centro educativo es aprovechado por los adolescentes y los puede impulsar para sus proyectos de vida.

\section{Conclusiones}

Si bien parece que la JEC no ha incluido los imaginarios posescolares de los alumnos peruanos en su implementación, las redes familiares de los migrantes andinos a Lima Sur han terminado siendo más trascendentales para el futuro de las próximas generaciones. Es importante reconocer estas prácticas no con categorías pasivas como herencia y tradición, sino con su fuerza movilizadora de aspiraciones (Appadurai, 2004). La política educativa en estudio podría seguir reproduciendo las brechas de la escuela en el Perú, pero ante ello se destaca el capital social, aunque la literatura también le encuentra límites (Cueto et al., 2005).

La permanencia de los lazos vecinales y familiares en los migrantes de segunda generación supone una excepción a la tendencia identificada por Golte y León (2011): los jóvenes limeños son menos marcados por el pasado rural que sus generaciones anteriores. La presente investigación corroboró que los escolares de la IE 7100 se siguen apoyando en las redes familiares para desenvolverse en una ciudad como Lima. Este tipo de prácticas permanecen para fines específicos, adquiriendo un carácter instrumental y clave en la configuración de sus vocaciones.

Este trabajo de campo registró que en dos colegios de Lima Sur hay una mayor aceptación de la vocación de servicio. Un sector de jóvenes peruanos está más interesado por las 
actividades terciarias antes que las agropecuarias o industriales. ¿Los adolescentes de hoy perciben que servir puede darles más ventajas que desventajas? ¿A cargo de quiénes quedaría el sector producción? Son preguntas interesantes para un análisis antropológico del trabajo en la sociedad peruana.

Además, es importante conocer los proyectos educativos del Estado para colegios con alumnado indígena y de familias campesinas como los Centros Rurales de Formación en Alternancia (CRFA). El objetivo de estas instituciones es el desarrollo de una producción local y la integración al mercado de sus egresados, pero también puede estar generando brechas en el intercambio con la empresa.

Se deben repensar las políticas pedagógicas teniendo en cuenta las aspiraciones de los estudiantes. Un colegio está recibiendo recursos públicos que pueden ser mejor aprovechados en otro. El Estado está destinando medio millón de soles en la IEE Ricardo Palma para un área curricular cuyos recursos pueden servir solo como un apoyo o en los ratos libres, es decir, para una instrucción que no forma parte de la vocación principal de los alumnos. Por el contrario, en colegios donde este tipo de formación es más útil para los escolares porque se dedicarán a ello al egresar, como la IE 7100, solo se dicta un curso de EPT.

En el Perú, metas como la implementación total de la JEC para el 2021, a fin de lograr el objetivo Economía, Competitividad y Empleo, uno de los que se propone el Plan Bicentenario, parecen responder más a una estrategia desarrollista que a enfocarse en garantizar las condiciones básicas de vida para que los futuros egresados de la escuela pública reciban una instrucción con la que potencien sus aptitudes y anhelos personales.

En concordancia con planteamientos de entidades de la esfera global, los esfuerzos por insertar a los adolescentes peruanos al mercado laboral demuestran que el Estado reconoce que ciertos sectores no podrían acceder a estudios posescolares. En un sentido similar, el BM muestra su preocupación por los jóvenes latinoamericanos que ni estudian ni trabajan.

Desde una antropología de las políticas públicas, se constató que el gobierno actual interpreta la educación como una acción colectiva de utilidad social, caracterizada por la inversión económica en cada estudiante. Esta lógica de costo-beneficio se distancia de la educación concebida como un derecho y, particularmente en el caso peruano, como una conquista de sectores emergentes y subalternos.

\section{Bibliografía}

Alcázar, Lorena (2016). Evaluación del diseño y proceso de implementación del modelo de Jornada Escolar Completa (JEC) para educación secundaria a nivel nacional. Lima: Proyecto Forge.

Anderson, Jeanine (1990). Sistemas de género e identidad de mujeres en culturas marcadas del Perú. Revista Peruana de Ciencias Sociales, 2 (1): 77-117.

Appadurai, Arjun (2004). The capacity to aspire. En Held, David y Henrietta Moore (eds.). Cultural politics in a global age (pp. 59-84). Oxford: One World.

Balarin, María, Lorena Alcázar, María Rodríguez y Cristina Glave (2017). Transiciones inciertas: una mirada a los jóvenes de contextos urbanos vulnerables de Lima. Lima: Grupo de Análisis para el Desarrollo.

Banco Mundial (1992). Educación técnica y formación profesional. Documento de política.

Banco Mundial (1996). Prioridades y estrategias para la educación. Examen del Banco Mundial.

Banco Mundial (2016). Ninis en América Latina. 20 millones de jóvenes en busca de oportunidades. 
Bonfiglio, Giovanni (2008). ¿Qué hay detrás de la baja aceptación de la educación técnica en el Perú? Debates de Sociología, (33): 77-94.

Bourdieu, Pierre y Jean-Claude Passeron (1964). Los herederos: los estudiantes y la cultura. Buenos Aires: Siglo XXI Editores.

Cotler, Julio (1968). La mecánica de la dominación interna y del cambio social en el Perú. En Matos Mar, José et al. Perú Problema: cinco ensayos (pp. 153-197). Lima: Moncloa Editores e Instituto de Estudios Peruanos.

Cueto, Santiago, Gabriela Guerrero, Juan León, Mary de Silva, Sharon Huttly, Mary E. Penny, Claudio F. Lanata y Eliana Villar (2005). Capital social y resultados educativos en el Perú urbano y rural. Documento de Trabajo $\mathrm{N}^{\circ}$ 28. Londres: Niños del Milenio.

Degregori, Carlos Iván (1986). Del mito del Inkarrí al mito del progreso: poblaciones andinas, cultura e identidad nacional. Socialismo y Participación, (36): 3-9.

Degregori, Carlos Iván, Cecilia Blondet y Nicolás Lynch (1986). Conquistadores de un nuevo mundo. De invasores a ciudadanos en San Martín de Porres. Lima: Instituto de Estudios Peruanos.

Franco, Ana y Hugo Nopo (2018). Ser joven en el Perú: educación y trabajo. Lima: Grupo de Análisis para el Desarrollo.

Golte, Jürgen y Doris León (2011). Polifacéticos: jóvenes limeños del siglo XXI. Lima: Atoq Editores e Instituto de Estudios Peruanos.

Golte, Jürgen y Norma Adams (1987). Los caballos de Troya de los invasores. Estrategias campesinas en la conquista de la gran Lima. Lima: Instituto de Estudios Peruanos.

Guerrero, Gabriela (2014). "Yo sé que va a ir más allá, va a continuar estudiando”: expectativas educativas de estudiantes, padres y docentes en zonas urbanas y rurales del Perú. Lima: Grupo de Análisis para el Desarrollo.

Guerrero, Gabriela, Claudia Sugimaru, Alexandra Cussianovich, Bieke De Fraine y Santiago Cueto (2016). Education aspirations among young people in Peru and their perceptions of barriers to higher education. Oxford: Young Lives.

Guzmán, María (2016). “En verdad es bien matado”. El papel de los profesores y tutores en la formación de estudiantes en un colegio preuniversitario en Villa El Salvador (Tesis de licenciatura). Lima: Pontificia Universidad Católica del Perú.

Huerta Morales, María (2013). Los múltiples sentidos de las políticas públicas: una propuesta etnográfica para su análisis. VII Jornadas Santiago Wallace de Investigación en Antropología Social. Sección de Antropología Social. Buenos Aires: Universidad de Buenos Aires.

INEI-Instituto Nacional de Estadística e Informática (2018). Perú: indicadores de Educación por departamento, 2007-2017. Lima: INEI.

Maldonado, Alma (2000). Los organismos internacionales y la educación en México. El caso de la educación superior y el Banco Mundial. Perfiles Educativos, 22 (87): 51-75.

Marcus, George (2001). Etnografía en/del sistema mundo. El surgimiento de la etnografía multilocal. Alteridades, 11 (22): 111-127.

Mead, Margaret (1962). Educación y cultura. Buenos Aires: Paidós.

Mead, Margaret (1977). Cultura y compromiso. Estudio sobre la ruptura generacional. Barcelona: Gedisa.

MIMP-Ministerio de la Mujer y Poblaciones Vulnerables (2018). Informe estadístico - Julio 2018. Violencia en cifras. Lima: MIMP.

Nandy, Ashis (2011). Imágenes del Estado. Cultura, violencia y desarrollo. México: Fondo de Cultura Económica.

Rojas, Vanessa y Alejandra Cussianovich (2013). Le va bien en la vida: Cambios y permanencias en el bienestar subjetivo de un grupo de adolescentes en Perú. Lima: Grupo de Análisis para el Desarrollo.

Shore, Cris y Susan Wright (1997). Policy: a new field of anthropology. En Shore, Cris y Susan Wright (eds.). Anthropology of Policy: critical perspectives on governance and power (pp. 3-39). Londres y Nueva York: Routledge. 
Urbano, Estefanía (2018). Lo que quiero ser: toma de decisiones respecto a la ocupación futura en adolescentes de dos instituciones educativas de Lima (Tesis de licenciatura). Lima: Pontificia Universidad Católica del Perú.

Wedel, Janine, Cris Shore, Gregory Feldman y Stacy Lathrop (2005). Toward an anthropology of public policy. The Annals of the American Academy Political and Social Science, vol. 600 (1): 30-51.

Wolf, Eric (1982). Europa y la gente sin historia. México: Fondo de Cultura Económica.

Presentado: 19 agosto 2019

Aceptado: 24 diciembre 2019

Publicado online: 15 julio 2020 